\title{
In control of switching, motion, and organization*
}

\author{
Ben. L. Feringa ${ }^{\ddagger}$, Richard A. van Delden, and Matthijs K. J. ter Wiel \\ Department of Organic Chemistry, Stratingh Institute, University of Groningen, \\ Nijenborgh 4, 9747 AG Groningen, The Netherlands
}

\begin{abstract}
Nature's solutions to control organization, switching, and linear and rotary motion are not only extremely elegant, but fascinating if one considers the design and synthesis of artificial molecular systems with such functions in order to add components to the nanotoolbox. The synthesis of chiroptical molecular switches and their application in the control of the organization of liquid crystalline materials is outlined. The first light-driven unidirectional molecular motor is described, and the second-generation motor as well as approaches to control the speed of the rotary motion are discussed.
\end{abstract}

It is hard to imagine daily life without machines, motors, and computers and a variety of other devices. The bottom-up construction of switches, motors, and ultimately machines and robotics of nanosize dimensions offers a formidable challenge to chemists. Its realization might have far-reaching consequences in view of the impact of their macroscopic counterparts in society. It is evident that the development of transistors and macroscopic engines were decisive events during the information technology and industrial revolution, respectively, and it is tempting to attribute a similar role for molecular switches and molecular motors in the nanotechnology era ahead of us [1].

In a recent article entitled "The Once and Future Nanomachine", G. M. Whitesides notes "among the promised fruits of nanotechnology, small machines have always stood out. Their attraction is straightforward" [2]. Inspired by the discovery of a steadily growing number of fascinating biomolecular motors, which are crucial for the functioning of living cells, the study of synthetic motors on a molecular scale is one of the most tantalizing areas of science at the intersection of chemistry, physics, and molecular biology [3]. Nature's solution to control organization, switching, and linear and rotary motion are not only extremely elegant, but particularly fascinating if one considers the design and synthesis of artificial molecular systems with such functions [4,5]. Among the most marvelous examples in Nature are the DNA/RNA replication system, the porphyrin arrays in the light-harvesting complex, the retinal cis-trans photoisomerization in the process of vision and the muscle linear and ATPase rotary motors [6].

Synthetic approaches toward artificial machinery have resulted in several molecular systems in which switching, translational or rotary motion, or a change in shape or assembly is controlled by means of chemical, electrochemical, photochemical, or thermal input [7]. For instance molecular propellers [8], brakes [9], switches [5a], turnstiles [10], ratchets [11], and shuttles [12] have been constructed. Catenanes and rotaxanes comprise a family of compounds that have been shown to be particularly useful to demonstrate functions reminiscent of molecular machines [13], like translational motion of a ring on a string in a rotaxane [7b,14], or circumrotation of two rings in a catenane [15]. Sauvage

\footnotetext{
*Plenary lecture presented at the $16^{\text {th }}$ International Conference on Physical Organic Chemistry (ICPOC-16): Structure and Mechanism in Organic Chemistry, San Diego, California, USA, 4-9 August 2002. Other presentations are published in this issue, pp. 541-630.

‡Corresponding author: E-mail: feringa@chem. rug.nl
} 
reported the contraction and stretching of a linear rotaxane dimer resembling a natural muscle at work [16]. Recently, Stoddart and Zink demonstrated the threading and dethreading of rotaxanes assembled on a surface [17]. Rotation in a bisporphyrinate double-decker complex in response to external electrochemical stimuli was accomplished by Aida [18], whereas Gaub reported the first single-molecular machine based on photoactive azodyes [19].

We focus on the design of molecular switches and molecular motors, and the dynamic control of molecular chirality is crucial in the functioning of these systems. The principles of a chiroptical molecular switch and the first light-induced unidirectional molecular motor are described. Applications as molecular trigger elements to control supramolecular organization are also discussed.

\section{CHIROPTICAL MOLECULAR SWITCHES}

The development of the molecular switches discussed here was inspired by the process of vision in which the basic chemical step is a light-induced cis-trans isomerization of an alkene group in a proteinbound retinal, resulting in a change from a bend to a linear shape of the molecule, which triggers a change in the protein and ultimately a signal to the brain (Fig. 1) [5a].

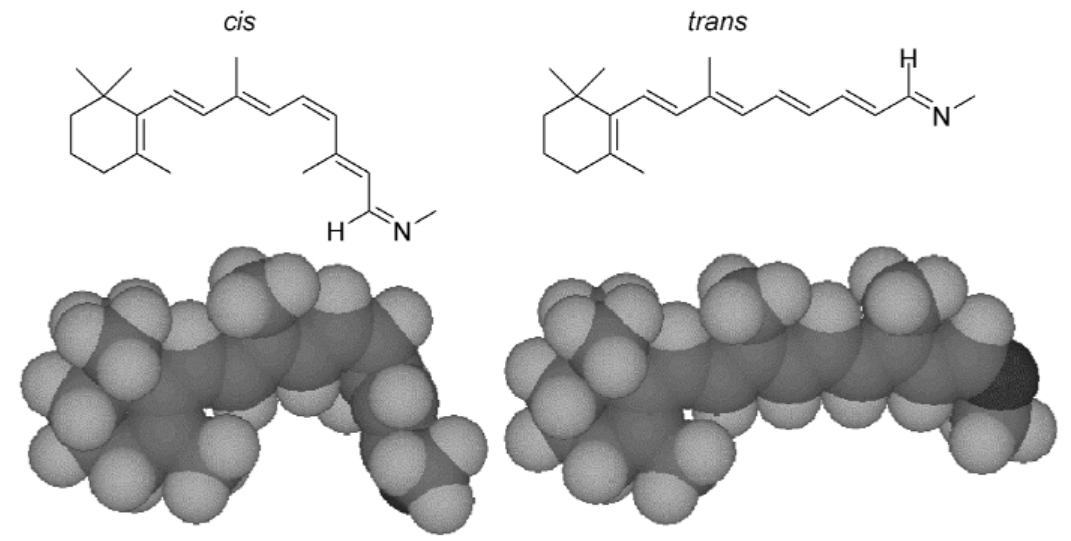

Fig. 1 Photoisomerization of protein-bound retinal in the process of vision.

In the synthetic chiroptical switches, a light-induced cis-trans isomerization is accompanied by a change in inherent helicity of the molecule. A major advantage of these chiroptical switches, compared to other photochromic compounds used as molecular memory or data-storage elements, is the possibility of nondestructive read-out of an optical recording system containing these materials. The change in optical rotation, for example, can simply be monitored at wavelengths remote from the switching wavelength. The principle of the chiroptical molecular switch is shown in Scheme 1.

The basic molecular structure is a helical-shaped unsymmetric, so-called sterically overcrowded, alkene 1 . The upper and lower half are connected by a central carbon-carbon double bond, which is considered the axis of rotation. Upon irradiation, the upper part turns from right to left relative to the lower part with a simultaneous change in helicity of the molecule. The left-handed $(M)$ and righthanded $(P)$ forms of these chiral molecules, which can be interconverted by light, represent two distinct states in a binary logic element [20]. 
<smiles>[W]</smiles>

(0)<smiles>CNc1ccc2c(c1)C(=C1CCSc3ccc4ccccc4c31)c1cc([N+](=O)[O-])ccc1S2</smiles>

(M)-cis-1a
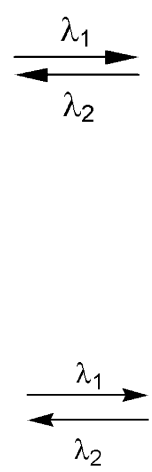

2<smiles>[C-]C1CC1</smiles>

(1)<smiles>CN1C=CC2=C(C1)c1cc([N+](=O)[O-])ccc1Sc1cc([N+](=O)[O-])ccc12</smiles>

(P)-trans-1b

Scheme 1

The molecular structure of (P)-cis-1a (Fig. 2) is illustrative for the antifolded helical shape of these chiral alkenes. In order to avoid unfavorable steric interactions at the "fjord region", these molecules adopt a helical shape; either a left-handed $(M)$-helix or a right-handed $(P)$-helix is present.

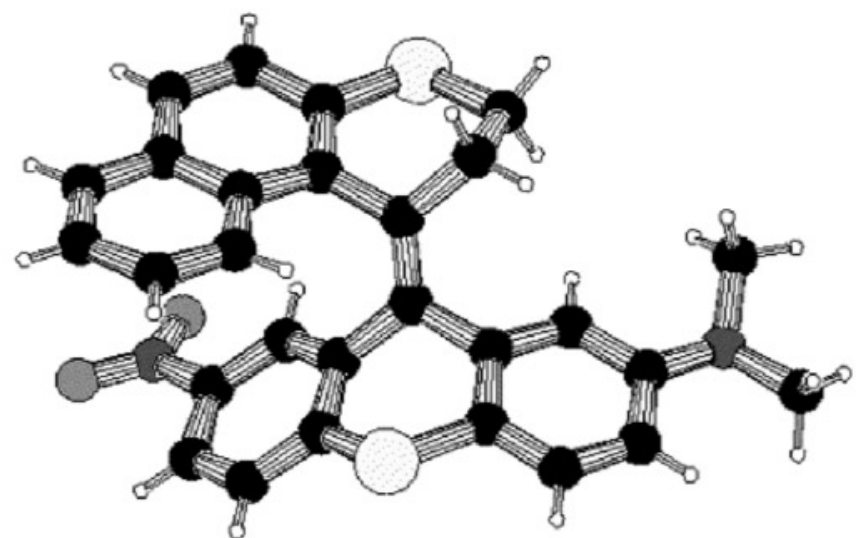

Fig. 2 Molecular structure of $(P)$-cis-1a.

The central double bond (the axis) has a normal bond length (1.353 $\AA$ ) and shows only slight deviation from planarity. Due to the presence of substituents in the lower half of these compounds, the cis-1a and trans-1b forms are pseudoenantiomers, and the overall helicity of the cis and trans forms is roughly mirror image. As a consequence, photochemical interconversion of the photobistable $M$ and $P$ forms can be accomplished at two different wavelengths $\lambda_{1}$ and $\lambda_{2}$ (Scheme 1) [21]. The introduction of a naphtho[2,1-b]thiopyran upper half and nitro-acceptor and dimethylamine-donor moieties in the lower half as in 1, resulted in remarkable enhanced stability and reversibility and a bathochromic shift in the absorption spectra compared to the earlier examples of chiroptical switches [21a]. Using 365-nm light, a $(M)$-1a / $(P)$-1b ratio of 30:70, and with 435-nm light a 90:10 ratio of $(M)-\mathbf{1 a} /(P)-\mathbf{1 b}$ was found. The photomodulation of chirality, as detected by CD spectroscopy, is shown in Fig. 3.

It should be emphasized that 1a is perfectly stable toward thermal racemization (helix inversion of $(M)$-cis-1a to $(P)$-cis-1b) under ambient conditions $\left(\Delta G_{\mathrm{rac}}=122.2 \mathrm{~kJ} \cdot \mathrm{mol}^{-1}\right)$. This allows switching near the visible leading to photostationary states with large differences in helicity, and simultaneously the electronic interaction between upper and lower half (due to the position of the substituents) is modulated [21b]. The difference in electron donor-acceptor interaction could be further enhanced when the 


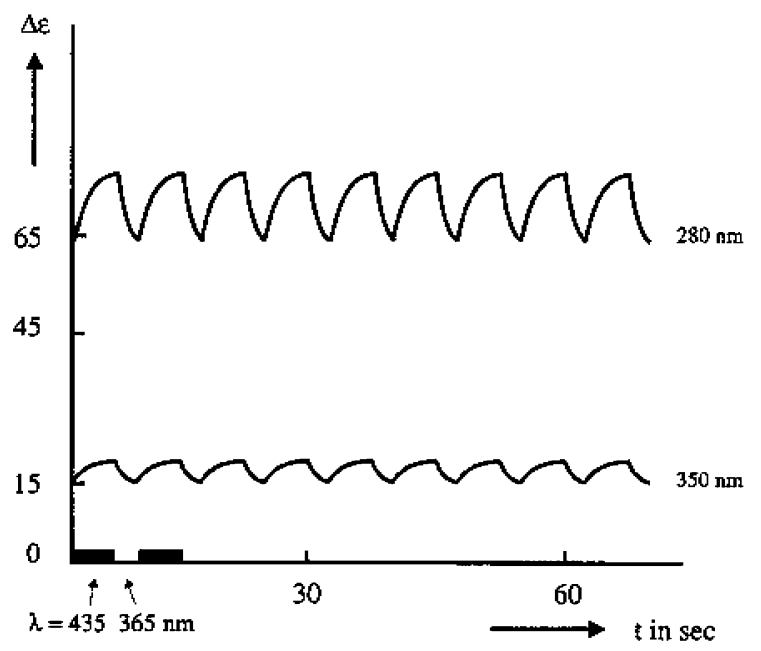

Fig. 3 Optical switching of $(M)$-cis-1a and $(P)$-trans-1b as monitored by CD spectroscopy.

dimethylamine donor group was moved to the upper half. These light-induced switching processes represent a controllable molecular motion with the benefit that the properties of these molecules can be readily tuned through modifications in substituents and structures. Furthermore, the reversible helicity change can be used for a digital molecular information storage system and the incorporation of these chiroptical switches as photoactive components in polymeric materials has been successful [20]. In addition, these systems have proven to be excellent trigger elements to control, via the light-induced motion and change in helicity, the mesoscopic organization and properties of liquid crystalline materials. Photochemical switching of LC phases using chiral photochromic guest molecules offers an alternative to current methods of addressing display materials [22]. Using a related helical-shaped photobistable alkene 2, switching between enantiomers at a single wavelength of light could also be accomplished, and the slight stereochemical bias that was found can be amplified in a liquid crystal film [23]. The switching process is shown in Scheme 2.<smiles>c1ccc2c(c1)Oc1ccc3ccccc3c1C2=C1c2ccccc2Sc2ccccc21</smiles>

$(P)-2$

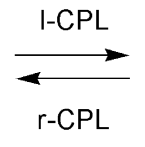

Scheme 2<smiles></smiles>

$(M)-2$

In this case, racemic $(P, M)-\mathbf{2}$ is irradiated at $313 \mathrm{~nm}$ alternating with left- or right-circular polarized light to generate a small excess $(0.07 \%$ ee) of $(M)$-2 or $(P)$-2, respectively. Photoderacemization also occurs upon irradiation (with 1- or d-CPL) of $(P, M)-2$ doped in nematic liquid crystalline phase of 4'-(pentyloxy)-4-biphenylcarbonitrile 3 to generate a cholesteric LC phase. Cholesteric phases with opposite screw sense (see Scheme 3) could be obtained simply by changing the handedness of the light. Furthermore, irradiation of these (chiral) cholesteric LC films with linearly polarized light at $313 \mathrm{~nm}$ resulted in a compensated nematic phase as a consequence of the formation of a 50:50 ratio of opposite helices of 3, e.g., photoracemization (Scheme 3). 


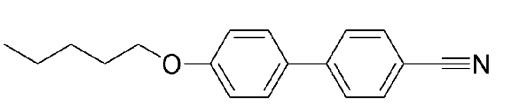

3

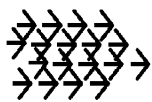

nematic

$50 \%(P)-2$

$50 \%(M)-2$
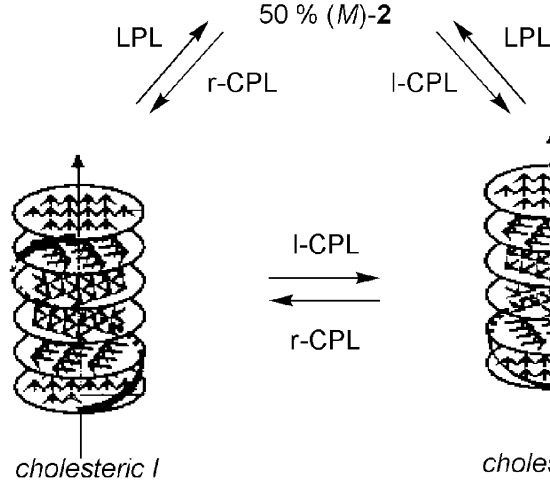

I-CPL

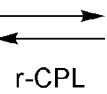

excess $(P)-2$

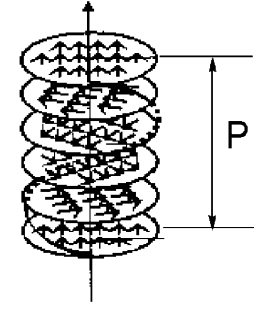

cholesteric II

excess $(M)-2$

Scheme 3

As is shown in Scheme 3, the photochemical switching processes of the LC phases are completely controlled by the changes in chirality of the light at a single $(313 \mathrm{~nm})$ wavelength. Furthermore, this system represents a chiral amplification method as the chirality of the circular polarized light is expressed in a small excess of one enantiomer of the molecular switch and subsequently amplified in the chirality of the helical organization in the induced cholesteric LC phase (Fig. 4).

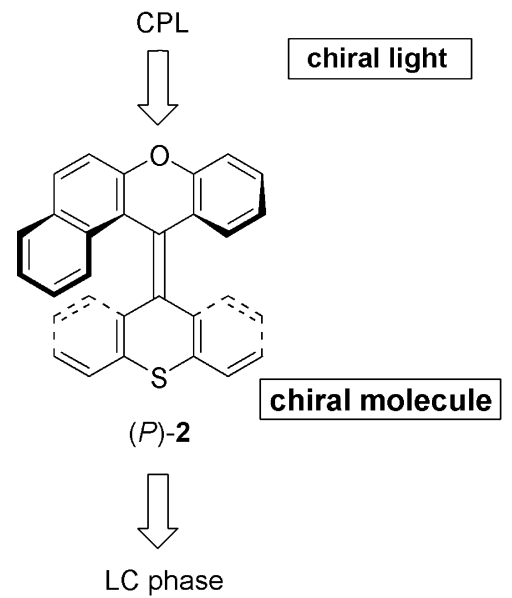

chiral supramolecular system

Fig. 4 Amplification of chirality.

Multifunctional molecular switches have also been developed in which the switching event is accompanied by a modulation of a second function. Successful examples include the use of molecular brakes to control the rotary speed of an attached propeller unit [8b], and the application in multimode switching of fluorescence [24]. 
It should be noted, in the context of the challenge to accomplish controlled motion at the molecular level that the relative direction of the movement in the chiroptical switches can be controlled by the wavelength of the light and depends only on the helicity of the molecule.

\section{MOLECULAR MOTORS}

The design and synthesis of molecular motors is one of the major endeavors in nanoscience. Inevitable motor functions will be needed to power nanomachines, although the design and functioning might be completely different from common macroscopic motors or biological motors.

We envisioned that three basic requirements must be satisfied in order to be able to construct a molecular motor: (i) repetitive $360^{\circ}$ rotary motion, (ii) consumption of energy, and (iii) unidirectional rotation. Recently, a chemical-driven [25] and a photochemical-driven [26] unidirectional rotary motor were reported simultaneously. Until these accomplishments, no synthetic system could be classified as a molecular motor, according to the prerequisites formulated above.

\section{First-generation motor}

The synthesis and photochemical and thermal isomerization processes of chiral overcrowded alkene $\left(3 R, 3^{\prime} R\right)-(P, P)$-trans-4a (Scheme 5) allowed us to realize the first light-powered molecular motor in which repetitive unidirectional $360^{\circ}$ rotary motion was achieved [26].

The exploitation of the following two fundamental principles were the key to success:

- $\quad$ photochemical trans-cis isomerization around a carbon-carbon double bond is usually a very fast and energetically uphill process in Nature;

- the concerted action of two chiral elements in a single chemical or physical event, by virtue of their diastereomeric nature, can lead to unique handedness.

In the structure of 4, two elements of chirality are present; a stereogenic center, with fixed stereochemistry, and a helical structure which can be changed by photoisomerization. Equally important are the two distinct orientations that can be adopted by the methyl substituents in these helical alkenes. The molecular structures and conformations of the stable and unstable forms of trans-4 are shown in Scheme 4.

In the more stable $\left(3 R, 3^{\prime} R\right)-(P, P)$-trans-4a, the methyl substituents adopt an axial orientation, whereas in the unstable $\left(3 R, 3^{\prime} R\right)-(M, M)$-trans-4d, the methyl substituents are in an equatorial orientation. MOPAC93-AM1 calculations show that the difference in stability $\left(35.9 \mathrm{~kJ} \cdot \mathrm{mol}^{-1}\right)$ is due to steric

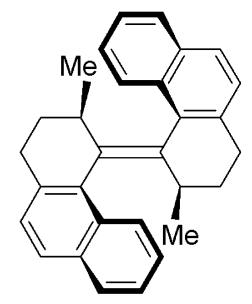

$\left(3 R, 3^{\prime} R\right)-(P, P)-$ trans-4a stable form (two axial methyl groups) $\Delta E=0.0 \mathrm{~kJ} \cdot \mathrm{mol}^{-1}$

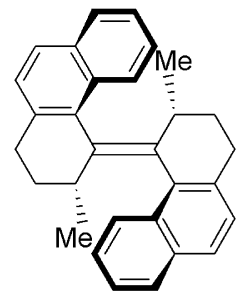

$\left(3 R, 3^{\prime} R\right)-(M, M)-$ trans-4d

less-stable form

(two equatorial methyl groups)

$\Delta E=+35.9 \mathrm{~kJ} \cdot \mathrm{mol}^{-1}$

Scheme 4 
hindrance of the methyl groups in the unfavorable equatorial orientation. For the cis-isomer, a similar situation is seen with a stable $\left(3 R, 3^{\prime} R\right)-(P, P)$-cis-4c form and an unstable $\left(3 R, 3^{\prime} R\right)-(M, M)$-cis-4d form with a difference in stability of $46.0 \mathrm{~kJ} \cdot \mathrm{mol}^{-1}$.

The photochemical and thermal isomerization processes of $\mathbf{4}$ are summarized in Scheme 5. Starting with $\left(3 R, 3^{\prime} R\right)-(P, P)$-trans- $\mathbf{4 a}$, the complete $360^{\circ}$ cycle includes four distinct steps; two reversible photochemical steps (steps 1 and 3), interconverting stable isomer trans-4a into unstable cis$\mathbf{4 b}$ and stable cis-4c into unstable trans-4d. Each photochemical step is followed by an irreversible thermal step (steps 2 and 4), converting the unstable cis- and trans- isomers in the stable forms. At temperature $>60{ }^{\circ} \mathrm{C}$, all isomerization processes occur, resulting in continuous $360^{\circ}$ rotary motion as long as the system is irradiated. The chirality at each state and the directionality of the rotary motion were assigned by $\mathrm{CD}$ spectroscopy.

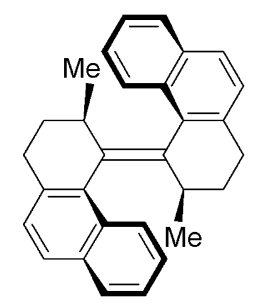

$\left(3 R, 3^{\prime} R\right)-(P, P)-$ trans-4a
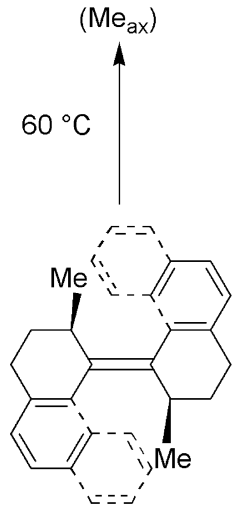

$\left(3 R, 3^{\prime} R\right)-(M, M)-$ trans-4d $\left(\mathrm{Me}_{\mathrm{eq}}\right)$
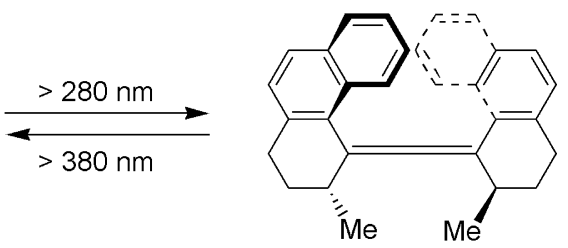

$\left(3 R, 3^{\prime} R\right)-(M, M)-c i s-4 b$

$\left(\mathrm{Me}_{\mathrm{eq}}\right)$

$20{ }^{\circ} \mathrm{C}$
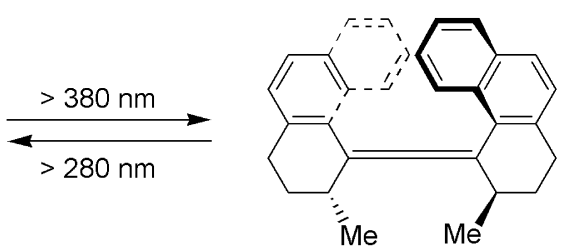

$\left(3 R, 3^{\prime} R\right)-(P, P)-$ cis-4c

$\left(\mathrm{Me}_{\mathrm{ax}}\right)$

Scheme 5

How does this motor function? Although light energy is necessary to power the molecular motor, the unidirectional rotation is governed by the methyl substituents. Photochemical trans-cis isomerization (step 1) of stable $\left(3 R, 3^{\prime} R\right)-(P, P)$-trans-4a to unstable $\left(3 R, 3^{\prime} R\right)-(M, M)-c i s-\mathbf{4 b}$ not only results in helix inversion, but forces the methyl substituents to adopt an unfavorable equatorial orientation. When the rotary motion in the molecule is continued in the same direction (step 2) by thermal interconversion into stable $\left(3 R, 3^{\prime} R\right)-(P, P)$-cis-4c, strain is released and the methyl substituents adopt again a more favorable axial orientation. In step 3 of the cycle, the photochemical isomerization of stable $\left(3 R, 3^{\prime} R\right)$ $(P, P)$-cis-4c into unstable $\left(3 R, 3^{\prime} R\right)-(M, M)$-trans-4d once again forces the methyl substituents in the unfavorable equatorial orientation. The final thermal step restores the original stable isomer $\left(3 R, 3^{\prime} R\right)-(P, P)$-trans-4a with the axial-oriented methyl group. The reverse of the thermal isomerization steps (4b to $\mathbf{4 c}$ and $\mathbf{4 d}$ to $\mathbf{4 a}$ ) are effectively blocked while the forward steps are possible due to light excitation (photoisomerization) of the molecule. One full cycle comprises four steps, where each lightdriven energetically uphill step is followed by a thermal energetically downhill step. When one-half of 
the molecule is considered the stator (Fig. 5), the other half (the rotor part) undergoes a repetitive $360^{\circ}$ rotation exclusively in a clockwise sense. The clockwise or counterclockwise rotation simply depends on the choice of enantiomer $[(P, P)-\mathbf{4}$ or $(M, M)-\mathbf{4}$, respectively] of the motor.
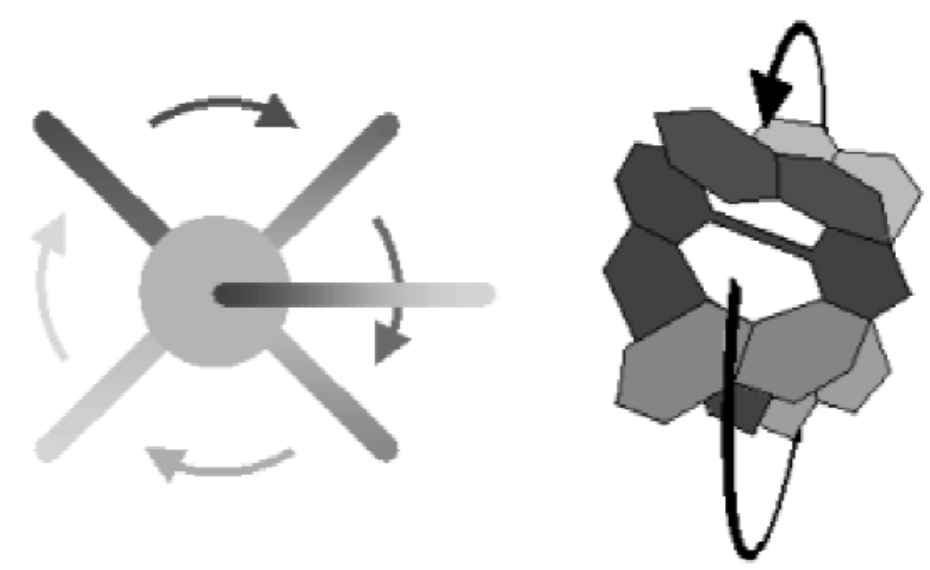

Fig. 5 Four-stage unidirectional rotary motion.

\section{Molecular motor at work}

With a successful molecular motor in hand, the next stage is to demonstrate that a macroscopic effect can be exerted by a motor functioning at the molecular level. Challenged by the idea that a mechanical effect (i.e., unidirectional rotation) can be used to change a property of a material or induced motion in ensembles of molecules, we embarked on the study of motors in liquid crystalline matrices [27]. As a first step toward a practical application of a motor function, we investigated whether the unidirectional molecular rotation that can be exerted by light can be amplified in the controlled movement of LC molecules to change the organization of the mesoscopic phase, and as a consequence, the chirality and macroscopic properties of the LC material. For this purpose, enantiomerically pure motor $\left(3 R, 3^{\prime} R\right)-(P, P)$-trans-4a was used as dopant $(6.16 \mathrm{wt} \%)$ in nematic LC material E7. Upon irradiation, the unidirectional rotary motion and simultaneous change in helicity induces a change in reflection wavelength of the LC film, which can be observed as a color change of the material from violet to red. In this way, the rotary motion at the molecular level can be observed by the color change of the film, as illustrated in Fig. 6.

A schematic representation of the changes at the molecular level, the organization of the LC host matrix, and the reflection wavelength of the light are given in Fig. 7. It is evident that the light-driven motion in the dopant induces the motion of a large ensemble of rod-like molecules during the reorganization in the LC film. This indirectly allows visual observation of the rotary motion. These findings not only demonstrate that a macroscopic effect, i.e., a change of the physical properties of a material, can be induced by a rotary molecular motor, but also that color pixels in an LC film can be generated using this supramolecular approach. The reflection wavelength can be readily tuned through the entire visible spectrum simply by changing the irradiation time. 


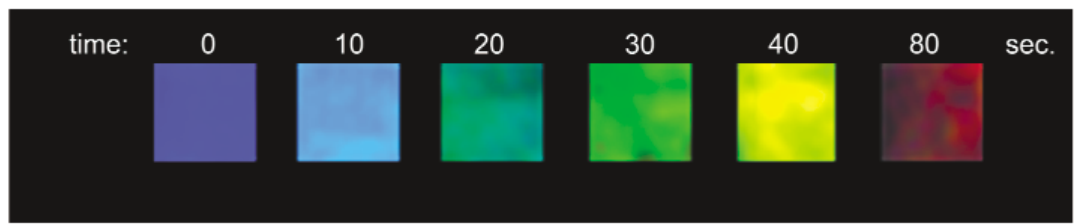

Fig. 6 Molecular motor induces a color change in an LC film.

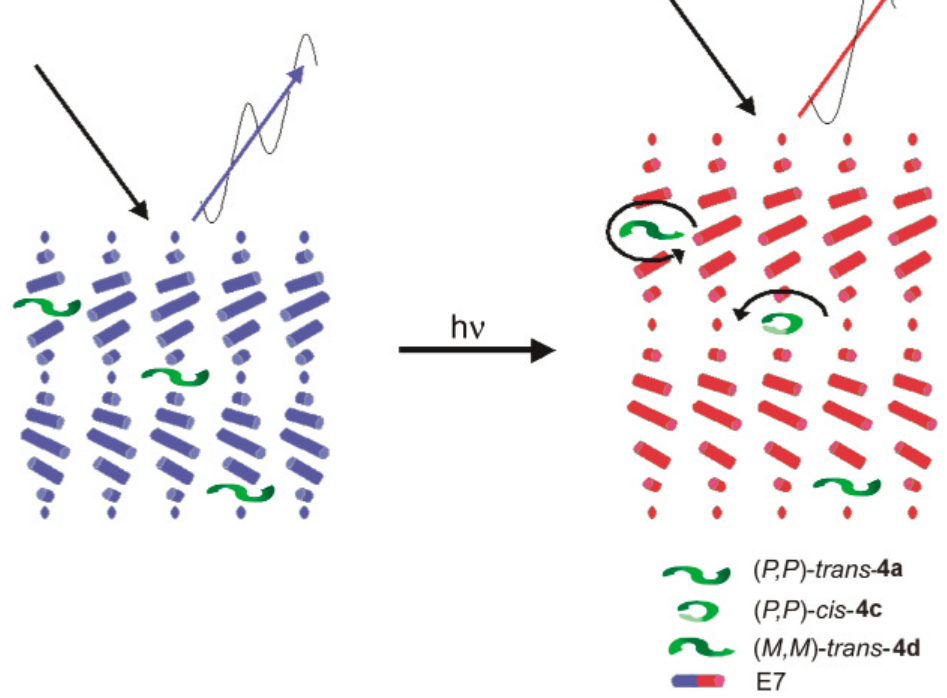

Fig. 7 Schematic representation of changes in doped LC film upon irradiation.

\section{Second-generation motor}

We reasoned that a redesign of our molecular motor would be necessary in the next stage toward multifunctional mechanical systems and in future attempts to use a molecular motor to power nanomachines. Motor 4, featuring identical upper and lower halves, was less suitable for such an endeavor. The second-generation motor has distinct upper and lower halves, in which the lower part can be used for connection to other molecules or surfaces, while the upper part still acts as a rotor as is illustrated in Fig. 8 [28].

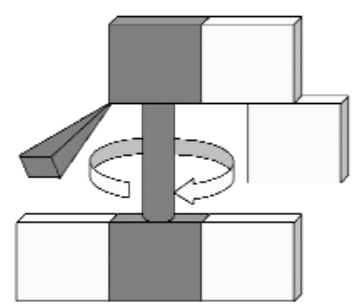<smiles>COc1ccc2c(c1)C(=C1c3ccccc3SCC1C)c1ccccc1S2</smiles>

$\left(2^{\prime} R\right)-(M)$-trans-5a

Fig. 8 Second-generation molecular motor. 
The second-generation motor 5 contains a $\left(2^{\prime} R\right)$-methyl-2, 3-dihydronaphthiopyran upper part and 2-methoxy-thioxanthene lower part. The molecular structure of stable $\left(2^{\prime} R\right)-(M)$-trans-5a, determined by X-ray analysis, shows that the methyl substituent again adopts the more favorable axial orientation. In contrast the structure of unstable $\left(2^{\prime} R\right)-(P)$-trans-5d features an equatorial orientation of the methyl group. Also, for the cis-isomer a stable $\left(2^{\prime} R\right)-(M)-c i s-5 \mathbf{c}$, axial methyl and unstable form $\left(2^{\prime} R\right)$ $(P)$-cis-5b, equatorial methyl are present. The different stereoisomers and the dynamic processes that are observed starting with $\left(2^{\prime} R\right)-(M)$-trans-5a are shown in Scheme 6.<smiles>COc1ccc2c(c1)/C(=C1/c3ccccc3SCC1C(C)C)c1ccccc1S2</smiles>

$\left(2^{\prime} R\right)-(M)-$ trans-5a<smiles>COc1ccc2c(c1)/C(=C1/c3ccccc3SC(C)C1C)c1cccc3cccc(c13)SCC2C</smiles>

$\left(2^{\prime} R\right)-(P)-$ trans-5d
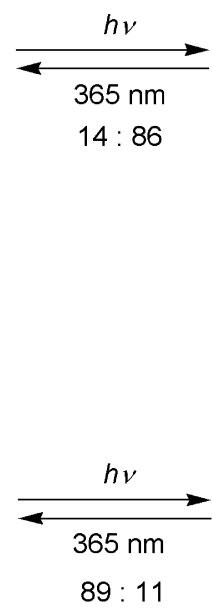

$89: 11$<smiles>COc1ccc2c(c1)/C(=C1\c3cc(OC)ccc3SCC1C)c1ccccc1S2</smiles>

$\left(2^{\prime} R\right)-(P)-c i s-5 b$<smiles>COc1ccc2c(c1)C(=C1c3ccccc3SC(C)C1[N+](=O)[O-])c1c(ccc3ccccc13)S2</smiles>

$\left(2^{\prime} R\right)-(M)-$ cis-5c

Scheme 6

Four distinct states are again observed, and two photochemical and two thermal steps add up to a full $360^{\circ}$ rotation cycle (Scheme 6). The distinct changes in helicity after each isomerization step can easily be monitored using CD spectroscopy, demonstrating the repetitive and unidirectional nature of the rotary motion that is induced. Once again, in the two thermal isomerization steps (steps 2 and 4 in Scheme 6), the $2^{\prime}$-methyl substituent in $\mathbf{5}$ adopts the more favorable axial orientation, and as a consequence the reverse rotation pathway is effectively blocked. Comparing this motor with the first-generation molecular motor (vide supra), it is remarkable that the presence of a single stereocenter and the change in orientation of one small methyl group is a sufficient condition to achieve unidirectional rotation.

\section{Increasing the speed of the rotary motion}

The rotary motion in these light-driven molecular motors is the result of two photochemical and two thermal isomerization steps. The rate of the rotary motion in a full $360^{\circ}$ rotation cycle is governed by the activation energy of the two thermal isomerization steps. The approach we followed to enhance the speed of the rotary motion involves lowering the activation energies of the thermal steps by the systematic modification of the bridging $\mathrm{X}$ and $\mathrm{Y}$ atoms in the upper and lower parts, respectively (Scheme 7).

The slow steps involve helix inversions in which arene moieties in upper and lower halves have to move along each other. A smaller bridging atom $\mathrm{X}$ or $\mathrm{Y}$ reduces the steric hindrance in the so-called "fjord region" of the molecule. This enables the upper and lower halves to slip along each other more easily. On the contrary, larger bridging atoms $\mathrm{X}$ and $\mathrm{Y}$ force the upper and lower halves toward each other increasing the barrier for passage. For instance, the presence of the smaller oxygen bridge $(\mathrm{Y}=\mathrm{O})$ 


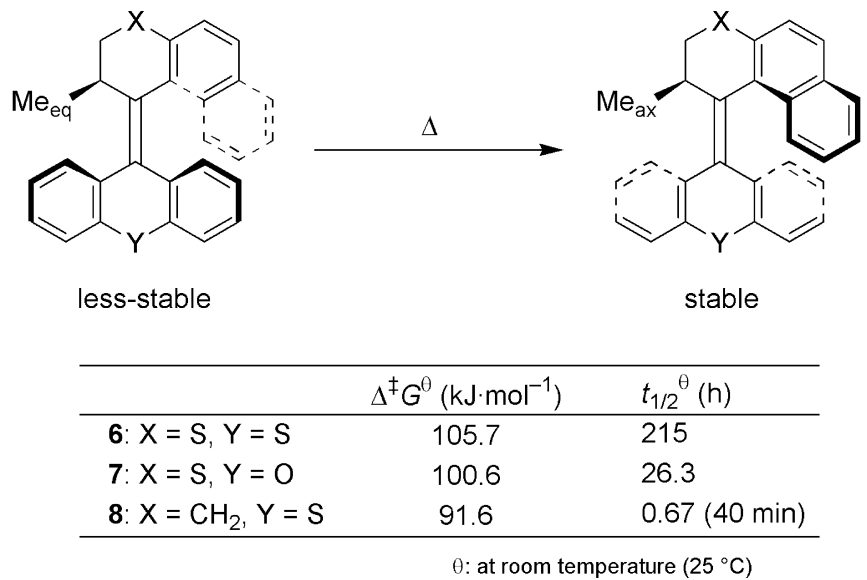

Scheme 7

in 7 compared to the sulfur bridge $(\mathrm{Y}=\mathrm{S})$ in $\mathbf{6}$ reduces the thermal barrier for helix inversion by $5.1 \mathrm{~kJ} \cdot \mathrm{mol}^{-1}$. A more pronounced effect is seen when $X$ is altered. By changing from $X=S(6)$ to $X=$ $\mathrm{CH}_{2}$ (8), the half-life for the thermal isomerization step could be decreased from $215 \mathrm{~h}$ to $40 \mathrm{~min}$ at room temperature [29].

Much to our surprise, in the motor systems 9 and 10, shown in Fig. 9, the rotary motions are slowed down when the naphthalene moiety in the upper part is replaced by the smaller benzene ring. This remarkable effect must be attributed to a higher ground-state energy of motor 9 [30]. It is evident that there is a delicate balance between ground-state distortion and steric and electronic effects on the photochemical and thermal isomerization processes in these sterically overcrowded alkenes.

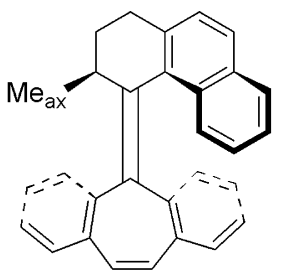

9

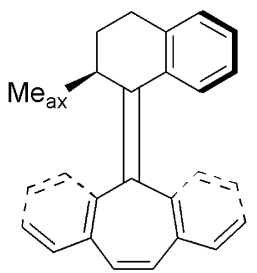

10

Fig. 9 Phenyl- vs. naphthyl-based molecular motor systems.

\section{CONCLUSIONS}

It has been shown that the dynamic control of chirality by light is an excellent means to achieve switching and motion and to accomplish modulation of supramolecular organization in materials. Chiroptical switches were constructed that can be used as molecular trigger elements or as reversible data storage units at the molecular level, allowing nondestructive readout. The first light-driven molecular motors were constructed, and the fundamental property of a molecular motor, i.e., energy consumption resulting in unidirectional rotary motion, was demonstrated by experiment. However, it is clear that these achievements are only a first step toward molecular information storage and retrieval systems and nanomechanical devices. Of the numerous challenges ahead, the construction of primitive nanoscale machines, powered by such motors, and the use of these motors to overcome Brownian motion, are particularly fascinating. 


\section{ACKNOWLEDGMENTS}

This research was financially supported by the Netherlands Foundation for Scientific Research NWO$\mathrm{CW}$. We are grateful to all students and coworkers who contributed to the molecular switch and motor project. A long-standing cooperation with Prof. N. Harada is gratefully acknowledged.

\section{REFERENCES}

1. (a) R. P. Feynman. In Miniaturization, H. D. Gilbert (Ed.), Reinhold, New York (1961); (b) Science 288, 79 (2000); special issue Movement: Molecular to Robotic; (c) E. Drexler. Nanosystems: Molecular Machinery, Manufacturing and Computation, Wiley, New York (1992).

2. G. M. Whitesides. In Scientific American, special issue Nanotech Sept., pp. 70-75 (2001).

3. M. Schliwa (Ed.). Molecular Motors, Wiley-VCH, Weinheim (2002).

4. R .D. Astumian. Sci. Am. 285, 44 (2001).

5. (a) B. L. Feringa (Ed.). Molecular Switches, Wiley-VCH, Weinheim (2001); (b) J.-P. Sauvage (Ed.). Molecular Machines and Motors Struct. Bond. 99 (2001).

6. D. S. Goodsell. Our Molecular Nature: The Body's Motors, Machines and Messages, Springer, New York (1996).

7. (a) Acc. Chem. Res. 34, 409 (2001), special issue Molecular Machines; (b) V. Balzani, M. GomezLopez, J. F. Stoddart. Acc. Chem. Res. 31, 405 (1998); (c) J.-P. Sauvage. Acc. Chem. Res. 31, 611 (1998); (d) B. L. Feringa. Acc. Chem. Res. 34, 504 (2001).

8. (a) K. Mislow. Chemtracts: Org. Chem. 2, 151 (1989); (b) A. M. Schoevaars, W. Kruizinga, R. W. J. Zijlstra, N. Veldman, A. L. Spek, B. L. Feringa. J. Org. Chem. 62, 4943 (1997).

9. T. R. Kelly, M. C. Boyer, K. V. Bhaskar, D. Bebbington, A. Garcia, F. Lang, M. H. Kim, M. P. A. Jette. J. Am. Chem. Soc. 116, 3657 (1994).

10. T. C. Bedard and J. S. Moore. J. Am. Chem. Soc. 117, 10662 (1995).

11. T. R. Kelly, I. Tellitu, J. P. Sestero. Angew. Chem., Int. Ed. 36, 1866 (1997).

12. (a) J.-P. Sauvage and C. Dietrich-Buchecker. Molecular Catenanes, Rotaxanes and Knots, WileyVCH, Weinheim (1999); (b) V. Balzani, A. Credi, F. M. Raymo, J. F. Stoddart. Angew. Chem., Int. Ed. 39, 3348 (2000).

13. A. R. Pease, J. O. Jeppesen, J. F. Stoddart, Y. Luo, C. P. Collier, J. R. Heath. Acc. Chem. Res. 34, 433 (2001).

14. (a) V. Bermudez, N. Capron, T. Gase, F. G. Gatti, F. Kajzar, D. A. Leigh, F. Zerbetto, S. Zhang. Nature 406, 608 (2000); (b) A. M. Brouwer, C. Frochot, F. G. Gatti, D. A. Leigh, L. Mollier, F. Paolucci, S. Rofia, G. W. H. Wurpel. Science 291, 2124 (2001).

15. F. M. Raymo and J. F. Stoddart. Switchable Catenanes and Molecular Shuttles in Molecular Switches, B. L. Feringa, (Ed.), p. 219, Wiley-VCH, Weinheim (2001).

16. (a) M. C. Jimenez, C. Dietrich-Buchecker, J.-P. Sauvage. Angew. Chem., Int. Ed. 39, 3284 (2000);

(b) B. L. Feringa. Nature 408, 151 (2002); News and Views, "In control of molecular motion".

17. S. Chia, J. Cao, J. F. Stoddart, J. I. Zink. Angew. Chem. Int. Ed. 40, 2447 (2001).

18. K. Tashiro, K. Knishi, T. Aida. J. Am. Chem. Soc. 122, 7921 (2000); see also [13b].

19. H. Gaub, T. Hugel, N. B. Holland, A. Cattani, L. Moroder, M. Seitz, H. E. Gaub. Science 296, $1102(2002)$.

20. B. L. Feringa, R. A. van Delden, N. Koumura, E. M. Geertsema. Chem. Rev. 100, 1789 (2000).

21. (a) B. L. Feringa, W. F. Jager, B. de Lange, E. W. Meijer. J. Am. Chem. Soc. 113, 5468 (1991); (b) W. F. Jager, J. C. de Jong, B. de Lange, N. P. M. Huck, A. Meetsma, B. L. Feringa. Angew. Chem., Int. Ed. 34, 348 (1995).

22. B. L. Feringa, N. P. M. Huck, H. A. van Doren. J. Am. Chem. Soc. 117, 9929 (1995).

23. N. P. M. Huck, W. F. Jager, B. de Lange, B. L. Feringa. Science 273, 1686 (1996).

24. N. P. M. Huck and B. L. Feringa. Chem. Commun. 1095 (1995). 
25. T. R. Kelly, H. DeSilva, R. A. Silva. Nature 401, 150 (1999).

26. (a) N. Koumura, R. W. J. Zijlstra, R. A. van Delden, N. Harada, B. L. Feringa. Nature 401, 152 (1999); (b) See also: A. P. Davis. Nature 401, 120 (1999); News and Views, "Synthetic molecular motors".

27. R. A. van Delden, N. Koumura, N. Harada, B. L. Feringa. Proc. Nat. Acad. Sci. USA 99, 4945 (2002).

28. N. Koumura, E. M. Geertsema, A. Meetsma, B. L. Feringa. J. Am. Chem. Soc. 122, 12005 (2000).

29. N. Koumura, E. M. Geertsema, B. L. Feringa. J. Am. Chem. Soc. 124, 5037 (2002).

30. E. M. Geertsema, N. Koumura, M. K. J. ter Wiel, A. Meetsma, B. L. Feringa. Chem. Commun. 2962 (2002). 